\title{
Regulação sanitária e desenvolvimento tecnológico: estratégias inovadoras para o acesso a medicamentos no SUS
}

\author{
Health regulation and technological development: \\ innovative strategies for accessing medicines in the SUS
}

Norberto Rech (https://orcid.org/0000-0003-4808-4277) ${ }^{1}$

Mareni Rocha Farias (https://orcid.org/0000-0002-4319-9318) ${ }^{1}$

${ }^{1}$ Departamento de Ciências Farmacêuticas, Centro de Ciências da Saúde, Universidade Federal de Santa Catarina. Campus Universitário Trindade Sala 72, Trindade. 88010-970 Florianópolis SC Brasil. norberto.rech@ufsc.br

\begin{abstract}
Regulatory science involves articulating knowledge that can establish the scientific bases for the definition of adequate and efficient regulatory mechanisms and practices. The interfaces between systemic and sectoral health and technological development policies were studied based on documentary analysis, especially from the National Pharmaceutical Policy (PNAF), with impacts on health regulation and stimulating the production of medicines of interest to the Unified Health System (SUS). The initiatives for the nationalized production of ARV "Efavirenz", which was the subject of a compulsory license in 2007, and the establishment of Partnerships for Productive Development (PDP), contributed to defining innovative regulatory frameworks and practices, emphasizing the Regulatory Technical Committees (CTR) for monitoring the internalization of technologies and health registration of the resulting products. The permeation capacity of the principles and strategic axes of the PNAF was identified in the sectoral policies that were analyzed. As of 2014, no macro or sectoral policies on expanding access to medicines in the SUS with impacts on regulations were identified.
\end{abstract}

Key words Pharmaceutical policy, Health surveillance, Medicines
Resumo A ciência regulatória é o campo de articulação de saberes que visa estabelecer as bases científicas para a definição de mecanismos e práticas regulatórias adequadas e eficientes. Com base na análise documental, foram estudadas as interfaces entre as politicas sistêmicas e setoriais no campo do desenvolvimento tecnológico e da saúde, especialmente a partir da Política Nacional de Assistência Farmacêutica (PNAF), com impactos no campo regulatório sanitário e no estímulo à produção de medicamentos de interesse do Sistema Único de Saúde (SUS). As iniciativas para a produção nacionalizada do medicamento antirretroviral "efavirenz", objeto de licença compulsória em 2007, bem como o estabelecimento das Parcerias para o Desenvolvimento Produtivo $(P D P)$, contribuíram para a definição de marcos e práticas regulatórias inovadoras, com destaque para os Comitês Técnico-Regulatórios (CTR) de acompanhamento das internalizações das tecnologias e registro sanitário dos produtos resultantes. Foi identificada a capacidade de permeação dos princípios e eixos estratégicos da PNAF no campo das politicas setoriais analisadas. A partir de 2014 não foram identificadas de macropolíticas ou políticas setoriais relacionadas à ampliação do acesso aos medicamentos no SUS com impactos no campo regulatório.

Palavras-chave Assistência farmacêutica, Vigilância sanitária, medicamentos 


\section{Introdução}

No que se refere à regulação sanitária e sua permeação no contexto das políticas públicas, o cenário brasileiro exige sua consideração à luz do processo de construção do direito à saúde, do Sistema Único de Saúde (SUS) e da formulação da Política Nacional de Saúde, a qual inclui a Política Nacional de Assistência Farmacêutica (PNAF) como um de seus componentes ${ }^{1}$, bem como de outras políticas setoriais.

No campo da saúde, as relações de produção e consumo de bens, serviços ou tecnologias são marcadas por suas amplas interfaces, especialmente naqueles setores que conformam o chamado Complexo Econômico-Industrial da Saúde (CEIS). Este complexo pode ser definido como um conjunto selecionado de atividades produtivas que mantêm relações intersetoriais de compra e venda de bens, serviços e/ou de conhecimentos e tecnologias, com clara relação de interdependência setorial ${ }^{2}$. Essas relações estão sujeitas às ações do Estado como promotor das possíveis interfaces e como regulador nas relações estabelecidas no contexto do CEIS ${ }^{3}$. Este complexo apresenta uma institucionalidade comum, representada pelos entes de regulação sanitária, de incorporação tecnológica, de ética em pesquisa, entre outros ${ }^{4}$.

O desenvolvimento de novas tecnologias e sua disponibilização para consumo global exige definições regulatórias que ultrapassam os limites dos países de origem ${ }^{5-7}$, gerando desafios constantes à ação dos entes regulatórios e dos Es$\operatorname{tados}^{8-10}$. Isto requer o fortalecimento dos sistemas regulatórios, os quais são componentes dos sistemas de saúde ${ }^{11,12}$.

Embora a expressão "Vigilância Sanitária" (VS) seja própria do Brasil, a regulação sanitária não diverge dos conceitos aceitos internacionalmente ${ }^{13,14}$, sendo que a dinâmica própria da vigilância sanitária está vinculada ao desenvolvimento científico e tecnológico e aos processos políticos que perpassam o Estado, o mercado e as sociedades. Nesse contexto, a ciência regulatória conforma o campo de conhecimento no qual a articulação e as interfaces entre distintos saberes possibilitam o desenvolvimento das bases científicas que possam ser empregadas para a garantia da segurança, qualidade e eficácia de produtos e serviços colocados à disposição das sociedades, bem como para o desenvolvimento de instrumentos e práticas que contribuam para a decisão e implantação de mecanismos de regulação ${ }^{15-17}$. Este campo do conhecimento tem sido foco de atenção de agências regulatórias reconhecidas mundialmente $e^{18,19}$.

O presente estudo teve como objetivo a identificação e análise de interfaces entre as políticas setoriais voltadas ao desenvolvimento tecnológico para a produção de medicamentos de interesse do Sistema Único de Saúde (SUS) e à regulação sanitária, tendo a PNAF como norteadora, na perspectiva de identificar ações e práticas regulatórias que possam contribuir para a redução de vulnerabilidades do SUS e para a sustentabilidade do acesso aos medicamentos.

\section{Métodos}

Trata-se de estudo realizado por meio de pesquisa documental, com adoção do modelo proposto por Walt e Gilson ${ }^{20}$ para a análise de políticas de saúde (Health Policy Analysis - HPA). O modelo analítico caracteriza-se como abrangente, permitindo a análise de relações complexas, ressaltando a inter-relação entre os quatro elementos constitutivos do chamado "triângulo de análise de política" e suas categorias: o contexto, o conteúdo, e o processo, para as quais podem contribuir diferentes atores. Para a análise de conteúdo das políticas foram empregadas as estratégias definidas no método do Planejamento Estratégico Situacional descrito por Matus ${ }^{21}$. A associação de distintas ferramentas analíticas é corroborada por diferentes autores ${ }^{22,23}$, o que pode contribuir para a maior solidez e abrangência das análises realizadas.

Em função do caráter polissêmico do termo "política", para o estudo adotou-se o entendimento de "política pública" enquanto processo no qual são elaborados e implementados programas de ação pública, contemplando dispositivos político-administrativos coordenados em torno de objetivos explícitos da ação governamental num setor ou espaço geográfico definido ${ }^{24}$.

O estudo compreendeu o período de 2003 a 2019, referente à formulação e aos 15 anos de implementação da PNAF. A busca de documentos foi realizada nos sítios eletrônicos dos ministérios da Saúde, da Economia e de Ciência, Tecnologia e Inovações, do Conselho Nacional de Saúde, da Agência Nacional de Vigilância Sanitária e do Banco Nacional de Desenvolvimento Econômico e Social e publicações em meio físico destas instituições.

Foram incluídas no estudo as ações públicas relacionadas ao desenvolvimento e internalização de tecnologias para a produção de medica- 
mentos de interesse do SUS, ao acesso e à regulação sanitária de medicamentos. Foram excluídos da análise os documentos que tratavam apenas de aspectos organizacionais e regramentos administrativos. A busca resultou na identificação de 212 documentos, lidos na íntegra e a aplicação dos critérios de inclusão e exclusão resultou em 110 documentos analisados.

\section{Resultados}

O Quadro 1 apresenta a síntese da análise quanto à tipologia e o conteúdo das políticas públicas com interfaces identificadas entre desenvolvimento tecnológico e regulação sanitária, no período do estudo.
A partir desses dados, foram analisados os contextos, os processos e os atores relacionados com as políticas referidas, cujos resultados estão apresentados no Quadro 2. As referências à regulação sanitária ou vigilância sanitária ocuparam lugar de destaque nas análises realizadas.

\section{Discussão}

A partir de 2003, o campo da assistência farmacêutica (AF) foi definido como uma das prioridades do Ministério da Saúde (MS), refletida na criação da Secretaria de Ciência, Tecnologia e Insumos Estratégico (SCTIE), do seu Departamento de Assistência Farmacêutica e Insumos Estratégicos (DAF) $)^{25,26}$ e na realização da $1^{\text {a }}$ Con-

Quadro 1. Síntese da análise de políticas públicas com interfaces entre desenvolvimento tecnológico na área de medicamentos e regulação sanitária, no período 2003-2019.

\begin{tabular}{|c|c|c|c|c|c|}
\hline \multirow{2}{*}{ Ano } & \multirow{2}{*}{$\begin{array}{c}\text { Macropolítica } \\
\text { (política } \\
\text { sistêmica) }\end{array}$} & \multirow{2}{*}{$\begin{array}{c}\text { Micropolítica } \\
\text { (política } \\
\text { setorial) }\end{array}$} & \multicolumn{3}{|c|}{ Conteúdo da política } \\
\hline & & & Situação real & Situação desejada & Ações \\
\hline $\begin{array}{l}2003- \\
2004\end{array}$ & $\begin{array}{l}\text { Formulação da } \\
\text { Política Nacional } \\
\text { de Assistência } \\
\text { Farmacêutica } \\
\text { (PNAF) }\end{array}$ & $\begin{array}{l}\text { Institucionalizar } \\
\text { a PNAF, nos } \\
\text { diferentes níveis } \\
\text { de gestão e } \\
\text { atuação do SUS }\end{array}$ & $\begin{array}{l}\text { Ações } \\
\text { fragmentadas no } \\
\text { âmbito da gestão } \\
\text { federal dos SUS } \\
\text { Dificuldades } \\
\text { de acesso dos } \\
\text { usuários a } \\
\text { medicamentos } \\
\text { no SUS } \\
\text { Desconexão } \\
\text { entre o acesso a } \\
\text { medicamentos } \\
\text { e as ações de } \\
\text { atenção à saúde }\end{array}$ & $\begin{array}{l}\text { Superação das } \\
\text { fragmentações das } \\
\text { ações envolvendo } \\
\text { planejamento, } \\
\text { aquisição, } \\
\text { distribuição } \\
\text { e acesso a } \\
\text { medicamentos no } \\
\text { SUS } \\
\text { O SUS como } \\
\text { orientador da } \\
\text { demanda de } \\
\text { medicamentos aos } \\
\text { setores produtivos } \\
\text { farmacêuticos, } \\
\text { com qualificação } \\
\text { do processo de } \\
\text { atenção à saúde }\end{array}$ & $\begin{array}{l}\text { Realização de } \\
\text { conferência nacional } \\
\text { temática e identificação } \\
\text { dos pressupostos da } \\
\text { PNAF } \\
\text { Definição e } \\
\text { institucionalização } \\
\text { da PNAF, com } \\
\text { características de } \\
\text { intersetorialidade e } \\
\text { norteamento de ouras } \\
\text { políticas } \\
\text { Qualificação da PNAF } \\
\text { como política estratégica } \\
\text { prioritária no âmbito da } \\
\text { gestão federal }\end{array}$ \\
\hline $\begin{array}{l}2003- \\
2006\end{array}$ & $\begin{array}{l}\text { Fortalecimento } \\
\text { da cadeia } \\
\text { produtiva } \\
\text { farmacêutica e do } \\
\text { desenvolvimento } \\
\text { tecnológico no } \\
\text { setor }\end{array}$ & $\begin{array}{l}\text { Inserir o } \\
\text { Ministério } \\
\text { da Saúde na } \\
\text { condução do } \\
\text { Fórum de } \\
\text { Competitividade } \\
\text { da Cadeia } \\
\text { Produtiva } \\
\text { Farmacêutica }\end{array}$ & $\begin{array}{l}\text { Forte retração } \\
\text { da indústria } \\
\text { brasileira de } \\
\text { fármacos e } \\
\text { cenário agravado } \\
\text { com a adoção } \\
\text { da Lei de } \\
\text { Propriedade } \\
\text { Industrial (1996) }\end{array}$ & $\begin{array}{l}\text { Estímulo ao } \\
\text { desenvolvimento } \\
\text { sustentável } \\
\text { do setor, com } \\
\text { agregação de } \\
\text { capacidades para a } \\
\text { internalização e o } \\
\text { desenvolvimento } \\
\text { de tecnologias de } \\
\text { interesse do SUS }\end{array}$ & $\begin{array}{l}\text { Identificação de } \\
\text { consensos para estímulo } \\
\text { ao desenvolvimento } \\
\text { dos setores produtivos } \\
\text { farmacêuticos (privado } \\
\text { e público) e inclusão } \\
\text { de Fármacos e } \\
\text { Medicamentos como } \\
\text { prioridades na Politica } \\
\text { Industrial, Tecnológica } \\
\text { e de Comércio Exterior } \\
\text { do Brasil }\end{array}$ \\
\hline
\end{tabular}


Quadro 1. Síntese da análise de políticas públicas com interfaces entre desenvolvimento tecnológico na área de medicamentos e regulação sanitária, no período 2003-2019.

\begin{tabular}{|c|c|c|c|c|c|}
\hline \multirow[b]{2}{*}{ Ano } & \multirow{2}{*}{$\begin{array}{c}\text { Macropolítica } \\
\text { (política } \\
\text { sistêmica) }\end{array}$} & \multirow{2}{*}{$\begin{array}{c}\text { Micropolítica } \\
\text { (política } \\
\text { setorial) }\end{array}$} & \multicolumn{3}{|c|}{ Conteúdo da política } \\
\hline & & & Situação real & Situação desejada & Ações \\
\hline 2004 & $\begin{array}{l}\text { Formulação da } \\
\text { Política Nacional } \\
\text { de Ciência, } \\
\text { Tecnologia e } \\
\text { Inovação em } \\
\text { Saúde (PNCTIS) }\end{array}$ & $\begin{array}{l}\text { Institucionalizar } \\
\text { a PNCTIS }\end{array}$ & $\begin{array}{l}\text { Incipiente } \\
\text { desenvolvimento } \\
\text { de atividades } \\
\text { de ciência, } \\
\text { tecnologia e } \\
\text { inovação nas } \\
\text { estruturas do } \\
\text { campo da saúde }\end{array}$ & $\begin{array}{l}\text { Desenvolvimento } \\
\text { nacional } \\
\text { sustentável, com } \\
\text { apoio na produção } \\
\text { de conhecimentos } \\
\text { ajustados às } \\
\text { necessidades do } \\
\text { país }\end{array}$ & $\begin{array}{l}\text { Realização da } 2^{a} \\
\text { Conferência Nacional } \\
\text { de Ciência, Tecnologia e } \\
\text { Inovação em Saúde } \\
\text { Definição e } \\
\text { institucionalização da } \\
\text { PNCTIS }\end{array}$ \\
\hline 2007 & \begin{tabular}{|l|} 
Redução de \\
vulnerabilidade \\
do Programa \\
Nacional de DST/ \\
Aids e garantia \\
do acesso a \\
medicamentos \\
antirretrovirais \\
no SUS \\
\end{tabular} & $\begin{array}{l}\text { Manutenção da } \\
\text { viabilidade de } \\
\text { financiamento } \\
\text { para a } \\
\text { aquisição de } \\
\text { medicamentos } \\
\text { antirretrovirais }\end{array}$ & $\begin{array}{l}\text { O custo do } \\
\text { medicamento } \\
\text { efavirenz agrava } \\
\text { a vulnerabilidade } \\
\text { do SUS e } \\
\text { negociações } \\
\text { infrutíferas } \\
\text { para redução de } \\
\text { preços }\end{array}$ & \begin{tabular}{|l|} 
Redução dos \\
custos de aquisição \\
do medicamento \\
efavirenz, com \\
redução da \\
vulnerabilidade \\
do Programa \\
Nacional de DST/ \\
Aids/SUS \\
\end{tabular} & $\begin{array}{l}\text { Adoção de Licença } \\
\text { Compulsória para uso } \\
\text { público e não comercial } \\
\text { das patentes do } \\
\text { medicamento efavirenz } \\
\text { para atendimento das } \\
\text { demandas do SUS }\end{array}$ \\
\hline 2008 & $\begin{array}{l}\text { Formulação } \\
\text { da Política de } \\
\text { Desenvolvimento } \\
\text { Produtivo }\end{array}$ & $\begin{array}{l}\text { CEIS como } \\
\text { programa } \\
\text { mobilizador da } \\
\text { área estratégica } \\
\text { da saúde } \\
\end{array}$ & $\begin{array}{l}\text { Pouco estímulo } \\
\text { às estruturas } \\
\text { produtivas em } \\
\text { saúde }\end{array}$ & \begin{tabular}{|l|} 
Incremento nos \\
investimentos \\
em pesquisa, \\
desenvolvimento e \\
inovação em saúde \\
\end{tabular} & $\begin{array}{l}\text { Inclusão do Complexo } \\
\text { Econômico-Industrial da } \\
\text { Saúde (CEIS) na política } \\
\text { de desenvolvimento }\end{array}$ \\
\hline 2009 & \begin{tabular}{|l|} 
Potencialização \\
do CEIS como \\
instrumento da \\
política industrial \\
brasileira
\end{tabular} & $\begin{array}{l}\text { Articulação } \\
\text { dos setores } \\
\text { produtivos face } \\
\text { às demandas do } \\
\text { SUS }\end{array}$ & $\begin{array}{l}\text { Demandas } \\
\text { crescentes } \\
\text { do SUS por } \\
\text { tecnologias e } \\
\text { elevação dos } \\
\text { gastos com } \\
\text { medicamentos }\end{array}$ & $\begin{array}{l}\text { Internalização } \\
\text { de tecnologias } \\
\text { de interesse do } \\
\text { SUS, com redução } \\
\text { da dependência } \\
\text { tecnológica, } \\
\text { redução de custos }\end{array}$ & $\begin{array}{l}\text { Adoção de "parcerias } \\
\text { para o desenvolvimento } \\
\text { produtivo" (PDP) como } \\
\text { estratégia para o emprego } \\
\text { do poder de compra do } \\
\text { Estado e indução positiva } \\
\text { do sistema produtivo em } \\
\text { saúde }\end{array}$ \\
\hline 2012 & $\begin{array}{l}\text { Fortalecimento } \\
\text { da indústria } \\
\text { nacional com } \\
\text { o aumento de } \\
\text { capacidade } \\
\text { produtiva, } \\
\text { inovadora e da } \\
\text { competitividade }\end{array}$ & $\begin{array}{l}\text { Estruturação e } \\
\text { modernização } \\
\text { da infraestrutura } \\
\text { pública de } \\
\text { tecnologia e } \\
\text { inovação em } \\
\text { saúde }\end{array}$ & $\begin{array}{l}\text { Dificuldades } \\
\text { estruturais e de } \\
\text { financiamento } \\
\text { para a } \\
\text { internalização } \\
\text { de tecnologia de } \\
\text { alto preço e de } \\
\text { grande impacto } \\
\text { sanitário }\end{array}$ & $\begin{array}{l}\text { Estímulo da } \\
\text { produção local de } \\
\text { produtos de alto } \\
\text { custo ou de grande } \\
\text { impacto sanitário } \\
\text { e social }\end{array}$ & $\begin{array}{l}\text { Fortalecimento dos } \\
\text { produtores públicos e a } \\
\text { ampliação de seu papel } \\
\text { de regulação de mercado, } \\
\text { com desenvolvimento } \\
\text { local de tecnologias } \\
\text { estratégicas para o SUS }\end{array}$ \\
\hline 2014 & $\begin{array}{l}\text { Racionalização } \\
\text { do poder de } \\
\text { compra do } \\
\text { Estado }\end{array}$ & $\begin{array}{l}\text { Redefinição } \\
\text { de diretrizes e } \\
\text { critérios para } \\
\text { a definição } \\
\text { de produtos } \\
\text { estratégicos } \\
\text { para o SUS e } \\
\text { estabelecimento } \\
\text { das PDP }\end{array}$ & $\begin{array}{l}\text { Necessidade de } \\
\text { melhoramento } \\
\text { do arcabouço } \\
\text { regulatório } \\
\text { das PDP e } \\
\text { do processo } \\
\text { de definição } \\
\text { de produtos } \\
\text { estratégicos }\end{array}$ & $\begin{array}{l}\text { Ampliar o acesso } \\
\text { da população } \\
\text { a produtos } \\
\text { estratégicos com } \\
\text { economicidade } \\
\text { e vantajosidade, } \\
\text { diminuindo a } \\
\text { vulnerabilidade } \\
\text { do SUS }\end{array}$ & $\begin{array}{l}\text { Revisão dos requisitos } \\
\text { para a definição de } \\
\text { produtos estratégicos } \\
\text { para o SUS e para } \\
\text { a formalização e } \\
\text { monitoramento das PDP }\end{array}$ \\
\hline
\end{tabular}

Fonte: Elaborado pelos autores, com base em modelos analíticos propostos por Walt e Gilson ${ }^{20}$ e Matus ${ }^{21}$. 
Quadro 2. Políticas públicas com interfaces com o desenvolvimento tecnológico na área de medicamentos, seus impactos no campo regulatório sanitário e atores envolvidos, no período 2003-2019.

\begin{tabular}{|c|c|c|c|c|}
\hline Ano & $\begin{array}{c}\text { Macropolítica } \\
\text { (política } \\
\text { sistêmica) }\end{array}$ & $\begin{array}{c}\text { Impacto na regulação } \\
\text { sanitária }\end{array}$ & Processos identificados & Atores envolvidos \\
\hline $\begin{array}{l}2003- \\
2004\end{array}$ & $\begin{array}{l}\text { Política nacional } \\
\text { de assistência } \\
\text { farmacêutica } \\
\text { (PNAF) }\end{array}$ & $\begin{array}{l}\text { Eixos estratégicos da } \\
\text { PNAF apontaram } \\
\text { a necessidade de } \\
\text { construção de uma } \\
\text { política de vigilância } \\
\text { sanitária para o acesso } \\
\text { da população a serviços } \\
\text { e produtos seguros, } \\
\text { eficazes e de qualidade }\end{array}$ & 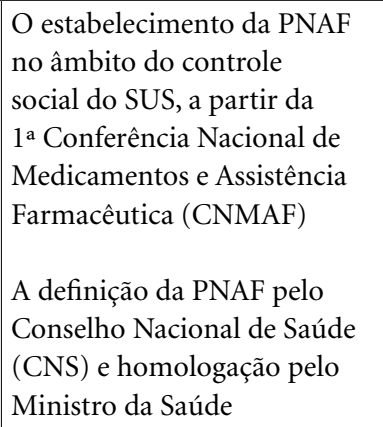 & $\begin{array}{l}\text { Conselho Nacional de } \\
\text { Saúde } \\
\text { Ministério da Saúde } \\
\text { Organização Pan- } \\
\text { Americana da Saúde } \\
\text { Delegados(as) } \\
\text { participantes da } 1^{\text {a }} \\
\text { CNMAF }\end{array}$ \\
\hline $\begin{array}{l}2003- \\
2006\end{array}$ & $\begin{array}{l}\text { Fortalecimento da } \\
\text { cadeia produtiva } \\
\text { farmacêutica e do } \\
\text { desenvolvimento } \\
\text { tecnológico no } \\
\text { setor }\end{array}$ & $\begin{array}{l}\text { Necessidade de que o } \\
\text { Sistema Nacional de } \\
\text { Vigilância Sanitária } \\
\text { (SNVS) adotasse } \\
\text { mecanismos para a } \\
\text { internalização das } \\
\text { diretrizes resultantes } \\
\text { dos consensos } \\
\text { obtidos no Fórum } \\
\text { de Competitividade } \\
\text { da Cadeia Produtiva } \\
\text { Farmacêutica }\end{array}$ & $\begin{array}{l}\text { Estabelecimento de } \\
\text { consensos no Fórum de } \\
\text { Competitividade da Cadeia } \\
\text { Produtiva Farmacêutica: } \\
\text { relevância econômica e } \\
\text { estratégica dos medicamentos } \\
\text { para o SUS; política industrial } \\
\text { farmacêutica como prioridade } \\
\text { de governo e política de } \\
\text { Estado } \\
\text { Inclusão de fármacose } \\
\text { medicamentos como uma das } \\
\text { quatro prioridades da Política } \\
\text { Industrial, Tecnológica e de } \\
\text { Comércio Exterior (PITCE) } \\
\text { do Brasil }\end{array}$ & $\begin{array}{l}\text { Representações } \\
\text { governamentais, } \\
\text { sob coordenação } \\
\text { dos Ministérios do } \\
\text { Desenvolvimento, } \\
\text { Indústria e Comércio e } \\
\text { da Saúde } \\
\text { Representações dos } \\
\text { segmentos produtivos. } \\
\text { Representações de } \\
\text { trabalhadores } \\
\text { Representações de } \\
\text { instituições de pesquisa }\end{array}$ \\
\hline 2004 & $\begin{array}{l}\text { Política Nacional } \\
\text { de Ciência, } \\
\text { Tecnologia e } \\
\text { Inovação em } \\
\text { Saúde (PNCTIS) }\end{array}$ & $\begin{array}{l}\text { Destaque para o papel } \\
\text { da Anvisa quanto à } \\
\text { anuência prévia para } \\
\text { patenteamento de } \\
\text { insumos para a saúde }\end{array}$ & $\begin{array}{l}\text { Estabelecimento da PNCTIS } \\
\text { se deu no âmbito do controle } \\
\text { social do SUS, baseado nas } \\
\text { definições da 2a Conferência } \\
\text { Nacional de Ciência, } \\
\text { Tecnologia e Inovação em } \\
\text { Saúde (CNCTIS) e aprovação } \\
\text { pelo Conselho Nacional de } \\
\text { Saúde }\end{array}$ & $\begin{array}{l}\begin{array}{l}\text { Conselho Nacional de } \\
\text { Saúde }\end{array} \\
\text { Ministérios da Saúde, } \\
\text { de Ciência e Tecnologia } \\
\text { e da Educação } \\
\text { Pesquisadores } \\
\text { Atores sociais } \\
\text { integrantes do controle } \\
\text { social do SUS }\end{array}$ \\
\hline
\end{tabular}

continua

ferência Nacional de Medicamentos e Assistência Farmacêutica $^{27,28}$.

A Política Nacional de Assistência Farmacêutica (PNAF) foi estabelecida no Conselho Nacional de Saúde (CNS) pela Resolução CNS nº $338 / 2004^{1}$, e homologada pelo ministro da Saú$\mathrm{de}^{29}$, consolidando as permeações entre acesso e uso racional de medicamentos com as políticas intersetoriais de desenvolvimento científico, tecnológico e industrial do Brasil $^{26,27}$.

A PNAF constituiu a primeira política pública formulada e instituída no âmbito do controle social do $\mathrm{SUS}^{30}$, assumindo papel estratégico para além do processo de atenção à saúde, ten- 
Quadro 2. Políticas públicas com interfaces com o desenvolvimento tecnológico na área de medicamentos, seus impactos no campo regulatório sanitário e atores envolvidos, no período 2003-2019.

\begin{tabular}{|c|c|c|c|c|}
\hline Ano & $\begin{array}{c}\text { Macropolítica } \\
\text { (política } \\
\text { sistêmica) }\end{array}$ & $\begin{array}{c}\text { Impacto na regulação } \\
\text { sanitária }\end{array}$ & Processos identificados & Atores envolvidos \\
\hline 2007 & $\begin{array}{l}\text { Redução de } \\
\text { vulnerabilidade } \\
\text { do Programa } \\
\text { Nacional de DST/ } \\
\text { Aids e garantia } \\
\text { do acesso a } \\
\text { medicamentos } \\
\text { antirretrovirais } \\
\text { no SUS }\end{array}$ & $\begin{array}{l}\text { Edição da Portaria } \mathrm{n}^{\circ} \\
\text { 583/2007/Anvisa, a } \\
\text { qual instituiu o Comitê } \\
\text { Técnico-Regulatório } \\
\text { (CTR) no âmbito da } \\
\text { Anvisa com a finalidade } \\
\text { de acompanhar o processo } \\
\text { de desenvolvimento, } \\
\text { produção e registro no } \\
\text { Brasil do medicamento } \\
\text { objeto do Decreto n. } \\
6.108, \text { de } 04 \text { de maio de } \\
2007\end{array}$ & $\begin{array}{l}\text { Declaração do governo } \\
\text { brasileiro quanto ao interesse } \\
\text { público dos direitos de patente } \\
\text { sobre o medicamento efavirenz, } \\
\text { para fins de concessão de } \\
\text { licença compulsória para uso } \\
\text { não comercial } \\
\text { Edição do Decreto } \\
\text { Presidencial n }{ }^{\circ} 6.108 / 2007, \\
\text { estabelecendo o licenciamento } \\
\text { compulsório do efavirenz } \\
\text { Edição da Portaria } \\
\text { Interministerial n }{ }^{\circ} 128 / 2008, \\
\text { a qual estabeleceu as diretrizes } \\
\text { para a contratação de fármacos } \\
\text { e medicamentos pelos órgãos } \\
\text { e entidades integrantes do } \\
\text { Sistema Único de Saúde }\end{array}$ & $\begin{array}{l}\text { Ministério da Saúde } \\
\text { Ministério da Justiça } \\
\text { Casa Civil da } \\
\text { Presidência da } \\
\text { República } \\
\text { Anvisa } \\
\text { Fundação Oswaldo } \\
\text { Cruz, incluindo a } \\
\text { direção do Instituto } \\
\text { de Tecnologia } \\
\text { em Fármacos - } \\
\text { Farmanguinhos } \\
\text { Organizações não- } \\
\text { governamentais } \\
\text { representativas dos } \\
\text { segmentos de pessoas } \\
\text { vivendo com HIV/Aids }\end{array}$ \\
\hline 2008 & $\begin{array}{l}\text { Política de } \\
\text { Desenvolvimento } \\
\text { Produtivo }\end{array}$ & $\begin{array}{l}\text { Grupo Executivo do } \\
\text { Complexo Industrial } \\
\text { da Saúde (GECIS) } \\
\text { responsável por ações } \\
\text { para garantia da } \\
\text { isonomia na regulação } \\
\text { sanitária, de apoio à } \\
\text { qualidade da produção } \\
\text { nacional, modernização } \\
\text { das ações de vigilância } \\
\text { sanitária, simplificação e } \\
\text { agilização dos processos } \\
\text { regulatórios }\end{array}$ & $\begin{array}{l}\text { Retomada e ampliação } \\
\text { da abrangência e da } \\
\text { profundidade da Política } \\
\text { Industrial, Tecnológica e de } \\
\text { Comércio Exterior (PITCE) } \\
\text { estabelecida em 2004, com a } \\
\text { definição do CEIS como uma } \\
\text { das suas prioridades } \\
\text { O ente nacional gestor do SUS } \\
\text { assume a função de condutor } \\
\text { das iniciativas intersetoriais } \\
\text { voltadas à regulação e } \\
\text { melhoria da eficiência do } \\
\text { CEIS, envolvendo os campos } \\
\text { industrial, econômico } \\
\text { e tecnológico visando o } \\
\text { atendimento das demandas } \\
\text { do SUS }\end{array}$ & $\begin{array}{l}\text { Ministério da Saúde. } \\
\text { Casa Civil da } \\
\text { Presidência da } \\
\text { República } \\
\text { Ministérios do } \\
\text { Desenvolvimento, } \\
\text { Indústria e Comércio; } \\
\text { da Fazenda; do } \\
\text { Planejamento; de } \\
\text { Relações Exteriores e } \\
\text { de Ciência, Tecnologia } \\
\text { e Inovação } \\
\text { Anvisa e outras } \\
\text { agências nacionais de } \\
\text { regulação e fomento }\end{array}$ \\
\hline
\end{tabular}

do como princípio o seu papel norteador para a formulação de outras politicas setoriais, com destaque para as políticas de medicamentos, de desenvolvimento industrial e de ciência e tecnologia. No que tange à regulação sanitária, a PNAF definiu como um dos seus eixos prioritários a construção de uma política de vigilância sanitá- ria voltada à garantia do acesso da população a serviços e produtos seguros, eficazes e com qualidade ${ }^{1}$. Esta construção ainda representa lacuna a ser preenchida, uma vez que o Brasil ainda não avançou no estabelecimento formal de uma política nacional de vigilância sanitária. Quanto aos medicamentos, a PNAF assume a função estraté- 
Quadro 2. Políticas públicas com interfaces com o desenvolvimento tecnológico na área de medicamentos, seus impactos no campo regulatório sanitário e atores envolvidos, no período 2003-2019.

\begin{tabular}{|c|c|c|c|c|}
\hline Ano & $\begin{array}{c}\text { Macropolítica } \\
\text { (política } \\
\text { sistêmica) }\end{array}$ & $\begin{array}{c}\text { Impacto na regulação } \\
\text { sanitária }\end{array}$ & Processos identificados & Atores envolvidos \\
\hline 2009 & $\begin{array}{l}\text { Potencialização } \\
\text { do CEIS como } \\
\text { instrumento da } \\
\text { política industrial } \\
\text { brasileira }\end{array}$ & $\begin{array}{l}\text { Edição da Resolução } \\
\text { da Diretoria Colegiada } \\
\text { da Anvisa (RDC) } n^{\circ} \\
02 / 2011 \text {, } \\
\text { instituindo Comitês } \\
\text { Técnico-Regulatórios } \\
\text { (CTR) para acompanhar } \\
\text { e monitorar as Parcerias } \\
\text { para o Desenvolvimento } \\
\text { Produtivo (PDP) }\end{array}$ & $\begin{array}{l}\text { Definição, pelo GECIS, das } \\
\text { PDP como mecanismos de } \\
\text { política industrial utilizados na } \\
\text { saúde e visando a internalização } \\
\text { da produção e a transferência } \\
\text { de tecnologia de medicamentos, } \\
\text { insumos farmacêuticos ativos e } \\
\text { produtos de interesse do SUS } \\
\text { PDP como instrumento } \\
\text { da política de saúde para } \\
\text { viabilizar o estímulo ao CEIS e } \\
\text { o atendimento das demandas } \\
\text { do SUS }\end{array}$ & $\begin{array}{l}\text { Corpo técnico e } \\
\text { diretivo da Anvisa } \\
\text { Representações } \\
\text { dos laboratórios } \\
\text { farmacêuticos públicos } \\
\text { Representações das } \\
\text { empresas privadas } \\
\text { integrantes das PDP } \\
\text { GECIS }\end{array}$ \\
\hline 2012 & $\begin{array}{l}\text { Fortalecimento da } \\
\text { indústria nacional } \\
\text { com o aumento } \\
\text { de capacidade } \\
\text { produtiva, } \\
\text { inovadora e da } \\
\text { competitividade }\end{array}$ & $\begin{array}{l}\text { Inclusão da Anvisa } \\
\text { no comitê gestor do } \\
\text { Programa para o } \\
\text { Desenvolvimento do } \\
\text { Complexo Industrial } \\
\text { da Saúde (PROCIS) e } \\
\text { edição da RDC da Anvisa } \\
\mathrm{n}^{\circ} \text { 50/2012, a qual dispôs } \\
\text { sobre os procedimentos } \\
\text { para registro de } \\
\text { produtos em processo } \\
\text { de desenvolvimento } \\
\text { ou transferência de } \\
\text { tecnologias objetos de } \\
\text { PDP público-público } \\
\text { ou público-privado de } \\
\text { interesse do SUS }\end{array}$ & $\begin{array}{l}\text { Incorporação do GECIS } \\
\text { pelo Plano Brasil Maior, } \\
\text { estabelecido em } 2011 \text { pelo } \\
\text { Decreto nº } 7.540 \\
\text { Instituição do Programa } \\
\text { para o Desenvolvimento } \\
\text { do Complexo Industrial da } \\
\text { Saúde (PROCIS), por meio } \\
\text { da Portaria do Ministério } \\
\text { da Saúde nº 506/2012, } \\
\text { voltado ao fortalecimento da } \\
\text { infraestrutura de produção e } \\
\text { inovação em saúde do setor } \\
\text { público } \\
\text { Definição das diretrizes } \\
\text { para o estabelecimento das } \\
\text { PDP, por meio da Portaria } \\
\text { do Ministério da Saúde }{ }^{\circ} \\
837 / 2012\end{array}$ & $\begin{array}{l}\text { Corpo técnico e } \\
\text { diretivo da Anvisa } \\
\text { Comitê Gestor do } \\
\text { PROCIS } \\
\text { Produtores públicos e } \\
\text { privados participantes } \\
\text { das PDP }\end{array}$ \\
\hline
\end{tabular}

gica de política sistêmica (macropolítica), tendo o SUS como orientador da demanda aos setores produtivos farmacêuticos, cuja capilaridade passou a influenciar a formulação ou a tomada de decisões no âmbito de outras políticas públicas sistêmicas e políticas setoriais (micropolíticas).

No período de 2003 a 2006 o MS atuou intensamente na coordenação compartilhada com o Ministério do Desenvolvimento, Indústria e Comércio Exterior do Fórum de Competitividade da Cadeia Produtiva Farmacêutica ${ }^{31}$, sendo que os atores envolvidos aprofundaram as discussões relativas ao desenvolvimento científico e tecnológico e industrial, tendo como perspectiva o atendimento das demandas do SUS. Em contexto marcado pela busca de consensos, o Fórum identificou a relevância econômica e estratégica dos medicamentos para o SUS, caracterizando a política industrial farmacêutica como "prioridade de governo" e "política de Estado" e recomendando a priorização de políticas públicas voltadas à modernização e à capacitação dos laboratórios farmacêuticos públicos para o desempenho de atividades de pesquisa e desenvolvimento (P\&D), 
Quadro 2. Políticas públicas com interfaces com o desenvolvimento tecnológico na área de medicamentos, seus impactos no campo regulatório sanitário e atores envolvidos, no período 2003-2019.

\begin{tabular}{|c|c|c|c|c|}
\hline Ano & $\begin{array}{c}\text { Macropolítica } \\
\text { (política } \\
\text { sistêmica) }\end{array}$ & $\begin{array}{c}\text { Impacto na regulação } \\
\text { sanitária }\end{array}$ & Processos identificados & Atores envolvidos \\
\hline 2014 & $\begin{array}{l}\text { Racionalização do } \\
\text { poder de compra } \\
\text { do Estado }\end{array}$ & $\begin{array}{l}\text { Edição da RDC } \\
\text { 43/2014/Anvisa: } \\
\text { Aprova o Regulamento } \\
\text { Técnico para os } \\
\text { registros concedidos } \\
\text { aos entes públicos ou } \\
\text { privados decorrentes } \\
\text { de processos de PDP } \\
\text { ou de transferências } \\
\text { de tecnologia visando } \\
\text { a internalização } \\
\text { da produção de } \\
\text { medicamentos } \\
\text { considerados } \\
\text { estrateìgicos pelo } \\
\text { Ministério da Saúde, as } \\
\text { condições de vinculação } \\
\text { ao registro do processo } \\
\text { matriz daquele registro } \\
\text { de medicamento objeto } \\
\text { de petição primária } \\
\text { clone, bem como os } \\
\text { procedimentos de pós- } \\
\text { registro e renovação de } \\
\text { registro respectivos }\end{array}$ & $\begin{array}{l}\text { O Plano Nacional de Saúde } \\
\text { (2012 -2015), compatibilizado } \\
\text { com o Plano Plurianual } \\
\text { Anual (PPA) e aprovado pelo } \\
\text { Conselho Nacional de Saúde, } \\
\text { estabeleceu, como uma das } \\
\text { suas } 16 \text { (dezesseis) diretrizes, } \\
\text { a diretriz de fortalecimento } \\
\text { do complexo produtivo } \\
\text { e de ciência, tecnologia e } \\
\text { inovação em saúde como } \\
\text { vetor estruturante da agenda } \\
\text { nacional de desenvolvimento } \\
\text { econômico, social e } \\
\text { sustentável, com redução de } \\
\text { vulnerabilidade do acesso à } \\
\text { saúde } \\
\text { Redefinição, pelo MS, das } \\
\text { diretrizes para transferência } \\
\text { e absorção de tecnologia, } \\
\text { aquisição de produtos } \\
\text { estratégicos para o SUS no } \\
\text { âmbito das PDP }\end{array}$ & $\begin{array}{l}\text { Corpo técnico e } \\
\text { diretivo da Anvisa } \\
\text { Ministério da Saúde }\end{array}$ \\
\hline
\end{tabular}

Fonte: Elaborado pelos autores, com base em modelos analíticos propostos por Walt e Gilson ${ }^{20} \mathrm{e} \mathrm{Matus}^{21}$.

bem como a implementação de parcerias público-privadas como mecanismo de indução à produção industrial no setor ${ }^{31}$. Estas definições estiveram alinhadas àquelas apontadas pela PNAF.

Como uma consequência relevante do Fórum, a inclusão do tema "fármacos e medicamentos" como uma das quatro prioridades da Política Industrial, Tecnológica e de Comércio Exterior (PITCE), representou avanço importante para a coordenação das políticas setoriais de suporte ao desenvolvimento dos setores produtivos da saú$\mathrm{de}^{32,33}$. Como resultado mais imediato de tal priorização, em abril de 2004 o Banco Nacional de Desenvolvimento Econômico e Social (BNDES) lançou o Programa de Apoio à Cadeia Farmacêutica (Profarma), com os objetivos iniciais de possibilitar investimentos para a adequação do parque farmacêutico nacional às novas exigências regulatórias e indução da atividade inovadora na cadeia farmacêutica ${ }^{34}$. Embora as recomendações do Fórum tenham indicado a necessidade de que o Sistema Nacional de Vigilância Sanitária (SNVS) adotasse mecanismos para a internalização das diretrizes resultantes dos consensos obtidos $^{31}$, até o final do ano de 2006 não foram identificadas ações concretas nesse sentido.

Em 2004, o desafio da formulação de uma Política Nacional de Ciência, Tecnologia e Inovação em Saúde (PNCTIS) encontrou contexto político-institucional favorável à retomada das discussões emanadas da $1^{\text {a }}$ Conferência temática realizada em $1994^{35}$, na medida em que a política de ciência e tecnologia em saúde constitui componente da Política Nacional e Saúde ${ }^{35,36}$. O estabelecimento da Secretaria de Ciência, Tecnologia e Insumos Estratégicos (SCTIE)/MS contribuiu para este cenário positivo. A 2a Conferência Nacional de Ciência, Tecnologia e Inovação em Saúde (CNCTIS), realizada em julho de 2004, resultou na proposição do texto consolidado da PNCTIS, o qual foi submetido à deliberação do $\mathrm{CNS}^{37}$. Muitos itens definidos pela PNCTIS man- 
tiveram relação inequívoca com as definições da PNAF, sendo que, em relação à regulação sanitária, a PNCTIS destacou o papel da Anvisa quanto à anuência prévia para patenteamento de insumos para a saúde ${ }^{37}$.

O instrumento de anuência prévia da Anvisa nos pedidos de concessão de patentes de produtos e processos farmacêuticos foi decorrente da introdução do art. 229-C à Lei de propriedade Intelectual (Lei $n^{\circ}$ 9.279/96) pela Lei $n^{\circ} 10.196$, de 14 de fevereiro de $2001^{38}$. A aplicação do disposto no art. 229-C foi objeto da Portaria Conjunta $n^{\circ} 1$, de abril de 2017, pactuada por Anvisa e Instituto Nacional de Propriedade Industrial $(\text { INPI })^{39}$. As definiçõos desta portaria limitam a aplicação do instituto da anuência prévia pela Anvisa, uma vez que esta poderá apresentar subsídios ao exame do INPI quanto aos critérios de patenteabilidade em pedidos de interesse para as politicas de medicamentos ou de assistência farmacêutica no âmbito do SUS, mas sem poder de veto quanto ao cumprimento desses critérios. Esta decisão seguiu caminho oposto àquele indicado pela PNCTIS ${ }^{37}$.

Em abril de 2007, após infrutíferas negociações com a empresa detentora da patente, o governo brasileiro declarou, de interesse público os direitos de patente sobre o efavirenz, para fins de concessão de licença compulsória para uso não comercial $^{40}$, o que foi seguido pela edição do Decreto Presidencial $n^{\circ} 6.108 / 2007$, estabelecendo o licenciamento compulsório do efaviren $z^{41}$. Isto ocorreu como resposta governamental ao contexto de elevada vulnerabilidade do Programa Nacional de DST/AIDS (PN DST/AIDS), estabelecido em $1986^{42,43,44}$ e cuja gratuidade do acesso aos medicamentos pelas pessoas vivendo com HIV foi estabelecido pela Lei $n^{\circ} 9.313$, de novembro de $1996^{45}$. Os impactos da Lei de Patentes brasileira ${ }^{46}$ e a elevação constante dos preços dos medicamentos antirretrovirais (ARV) passaram a afetar a sustentabilidade do programa brasileiro, sendo que no período de 2006 e 2007 cerca de $70 \%$ do orçamento do PN DST/AIDS estavam comprometidos com a aquisição de medicamentos ARV importados ${ }^{47}$. Após a adoção da licença compulsória, a produção nacional do medicamento efavirenz foi assumida pelo Instituto de Tecnologia em Fármacos Farmanguinhos/ Fiocruz ${ }^{4,48}$, sendo viabilizada por parcerias com empresas farmacêuticas e farmoquímicas nacionais. Esta iniciativa foi ancorada na Portaria Interministerial $\mathrm{n}^{\circ} 128 / 2008$, a qual estabeleceu as diretrizes para a contratação de fármacos e medicamentos pelos órgãos e entidades integrantes do
Sistema Único de Saúde ${ }^{49,50}$. Este arranjo produtivo constituiu um dos exemplos pioneiros do uso da estratégia que mais tarde viria a configurar as Parcerias para o Desenvolvimento Produtivo $(\mathrm{PDP})^{4}$. Estas ações estiveram em consonância com os princípios e eixos estratégicos da PNAF ${ }^{1}$.

Nesse cenário, a Anvisa atuou proativamente e editou a Portaria $n^{\circ} 583 / 2007$, a qual instituiu o Comitê Técnico-Regulatório (CTR) no âmbito da Anvisa com a finalidade de acompanhar o processo de desenvolvimento, produção e registro no Brasil do medicamento objeto do Decreto n. 6.108, de 04 de maio de 200751. Esta definição foi inovadora no cenário regulatório-sanitário brasileiro, possibilitando interface precoce e colaborativa da autoridade regulatória com os entes produtivos responsáveis por iniciativas de desenvolvimento tecnológico de interesse nacional. Em fevereiro de 2009, Farmanguinhos/Fiocruz entregou ao MS os primeiros lotes da produção nacionalizada do ARV efavirez ${ }^{52}$, o que foi possível pela redução dos tempos regulatórios promovida pelo CTR.

Em maio de 2008, como uma das iniciativas de enfrentamento à marcada aceleração do déficit na balança comercial brasileira nos segmentos de fármacos e medicamentos desde os anos $2000^{53}$, foi instituída a Política Nacional de Desenvolvimento Produtivo. Esta política definiu o Complexo Econômico-Industrial da Saúde (CEIS) como uma das suas prioridades, o que foi acompanhado pela criação do Grupo Executivo para o Complexo Industrial da Saúde (GECIS), por meio do Decreto/2008 54,55 . O MS, como gestor nacional do SUS, assumiu a função de condutor das iniciativas intersetoriais voltadas à regulação e melhoria da eficiência do CEIS, envolvendo os campos industrial, econômico e tecnológico e visando o atendimento das demandas do SUS ${ }^{4,56}$. A política de desenvolvimento definida em 2008 retomou e ampliou tanto a abrangência como a profundidade da PITCE estabelecida em $2004^{57}$. Tal como previsto no Decreto de sua criação ${ }^{55}$, foi definida a criação de um fórum permanente de articulação com a sociedade civil para o assessoramento do GECIS, o que colocou a premissa de participação dos distintos atores sociais no processo de proposição de estratégias e ações para o desenvolvimento voltado à área da saúde ${ }^{58}$. Em relação à regulação sanitária, as competências do GECIS envolveram ações para a garantia da isonomia na regulação sanitária e de apoio à qualidade da produção nacional, incluindo a modernização das ações de vigilância sanitária; bem como o estabelecimento de uma rede de suporte à qualidade e competitividade da produção local e a 
simplificação e agilização dos processos regulatórios $^{36,53}$. Estas definições reforçaram as iniciativas da Anvisa para a adoção dos CTR como prática regulatória, especialmente a partir dos resultados positivos observados no processo decorrente da licença compulsória adotada em 2007.

No cenário em que as políticas públicas no campo da saúde foram direcionadas para uma articulação virtuosa entre a atenção à saúde e o desenvolvimento industrial ${ }^{59}$, as compras públicas se tornaram um importante instrumento indutor de capacitação tecnológica e desenvolvimento da base produtiva, visando reduzir a vulnerabilidade do SUS e a geração de investimentos, emprego e renda ${ }^{4}$. Estes pressupostos estão em consonância com as diretrizes e os eixos estratégicos estabelecidos na PNAF. A partir de 2009, as PDP foram definidas como instrumento estabelecido no âmbito da política de saúde para viabilizar o estímulo ao CEIS e atendimento das demandas do SUS $4,36,60,61$. Constituem, portanto, mecanismo de política industrial utilizada na saúde visando a internalização da produção e a transferência de tecnologia de medicamentos, insumos farmacêuticos ativos e produtos de interesse do SUS ${ }^{62}$. Esta iniciativa, sob coordenação do MS e apresentada no âmbito do GECIS em $2009^{61}$, se dá pelo estabelecimento de parceria entre a empresa detentora da tecnologia e um ente produtor público qualificado para o fornecimento do produto ao SUS durante o período de absorção tecnológica, sendo a aquisição centralizada pelo $\mathrm{MS}^{4,63,64}$. Esta estratégia deu consequência prática da inserção do CEIS entre os eixos estratégicos do planejamento nacional em saúde, contribuindo para o fortalecimento da indústria farmoquímica nacional e dos laboratórios farmacêuticos oficiais, a partir de arranjos de transferências de tecnologias demandadas pelo SUS ${ }^{28}$. No processo de construção e implantação das PDP, o MS, especialmente por meio da SCTIE, assumiu protagonismo destacado como ator institucional, tendo obtido resultados importantes para a sustentabilidade do acesso aos medicamentos no SUS. No período de 2011 ao início de 2017, a economia resultante das aquisições centralizadas de medicamentos objetos das PDP atingiu a ordem de $\mathrm{R} \$ 4,68$ bilhões $^{36}$. Nesse contexto, a Anvisa definiu pela instituição de Comitês Técnico-Regulatórios (CTR) para acompanhar e monitorar as diferentes parcerias definidas para cada um dos laboratórios farmacêuticos públicos envolvidos ${ }^{61,63}$. Tais CTR encontraram ancoramento formal na Resolução da Diretoria Colegiada da (RDC) da Anvisa de $n^{\circ} 02 / 2011^{65}$, tendo como escopo a experiência exitosa da prática regulatória adotada quando da licença compulsória do efavirenz em 2007.

As ações de inclusão do CEIS na política nacional de desenvolvimento e seu fortalecimento orientado ao atendimento das demandas do SUS encontraram reforço com a incorporação do GECIS pelo Plano Brasil Maior, estabelecido em 2011 pelo Decreto $n^{\circ} 7.540^{66,67}$. Nesse novo cenário, o MS instituiu o Programa para o Desenvolvimento do Complexo Industrial da Saúde (PROCIS), por meio da Portaria MS $n^{\circ} 506 / 2012^{68}$, voltado ao fortalecimento da infraestrutura de produção e inovação em saúde do setor público. Nesse contexto foram definidas as diretrizes para o estabelecimento das PDP, por intermédio da Portaria MS n ${ }^{\circ} 837 / 2012^{69}$, a qual esteve alicerçada no Plano Nacional de Saúde ${ }^{70}$.

As interfaces do PROCIS com a vigilância sanitária estão demonstradas pela inclusão da Anvisa no seu comitê gestor ${ }^{68}$ e pelas ações desta agência voltadas ao desenvolvimento tecnológico e industrial, tal como a edição da RDC n ${ }^{\circ}$ 50/2012, a qual dispôs sobre os procedimentos para registro de produtos em processo de desenvolvimento ou transferência de tecnologias objetos de Parcerias de Desenvolvimento Produtivo público-público ou público-privado de interesse do Sistema Único de Saúde ${ }^{71}$. Esta definição regulatório-sanitária foi inovadora ao instituir mecanismos para a sistemática internalização de todas as informações relativas ao desenvolvimento dos medicamentos objetos de PDP, resultando na composição paulatina e monitorada dos dossiês dos produtos, com redução dos tempos regulatórios exigidos para a análise e definição sobre os respectivos registros e aceleração da sua disponibilização no SUS.

A revisão do marco legal de sustentação das PDP foi definida pela Portaria MS ${ }^{\circ}$ $2.531 / 2014^{72}$. Este normativo está relacionado à macropolítica de uso e racionalização do poder de compra do Estado, incluindo os critérios para a definição de produtos estratégicos para o SUS e o estímulo ao desenvolvimento do $\mathrm{CEIS}^{73}$. A regulamentação do uso do poder de compra do Estado foi objeto da Política Nacional de Inovação Tecnológica na Saúde (PNITS), definida pelo Decreto $\mathrm{n}^{\circ} 9.245 / 2017^{74}$, já num contexto de fragilização da democracia brasileira promovido pelo golpe jurídico-parlamentar ocorrido em $2016^{75}$. Todavia, a PNITS não agregou qualquer avanço no tocante à regulação sanitária. $\mathrm{O}$ contexto no qual se deu a revisão da regulamentação das PDP foi marcado pela necessidade de maior transparência dos processos para sua definição e busca de maior segurança jurídica para a tomada de de- 
cisões no âmbito da gestão pública. As iniciativas adotadas foram fortemente influenciadas pelas recomendações resultantes da auditoria realizada pelo Tribunal de Contas da União (TCU), a qual avaliou a regularidade das PDP firmadas pelo $\mathrm{MS}^{76}$. O novo marco legal representou evolução quanto aos critérios para a definição de produtos estratégicos para o SUS estabelecidos em 2008 pela Portaria MS n ${ }^{\circ} 978^{77}$. Entretanto, estudo recente indica que os critérios adotados para a construção das listas de produtos estratégicos para o SUS não incorporam elementos da avaliação de tecnologias em saúde e do uso de evidências, sendo que o processo carece de interações entre pesquisadores e tomadores de decisão ${ }^{78,79}$. No que se refere à regulação sanitária, a revisão do norteamento legal das PDP reafirmou o papel dos CTR e incluiu a Anvisa nas Comissões Técnicas de Avaliação (CTA), responsáveis pela análise e avaliação das propostas de PDP. Tais definições reforçam o papel estratégico da Anvisa no contexto da política de estímulo ao CEIS e o entendimento dos CTR como modelo de prática no campo regulatório-sanitário.

\section{Considerações finais}

O estudo identificou interfaces importantes entre a evolução dos marcos e práticas regulatórias com as iniciativas de estímulo ao desenvolvimento tecnológico para a produção nacional de medicamentos de interesse do SUS, a partir dos norteamentos da Política Nacional de Assistência Farmacêutica (PNAF).

A instituição das PDP constituiu estratégia importante para a redução das vulnerabilidades do SUS e concretizou os Comitês Técnico-Regulatórios (CTR) como prática inovadora no contexto da vigilância sanitária, considerando a experiência exitosa realizada quando da licença compulsória para a produção nacional do medicamento efavirenz em 2007. Os estímulos ao CEIS também impulsionaram a adoção de novos marcos e novas práticas regulatórias relacionados à internalização e ao desenvolvimento de tecnologias, mantendo interfaces com a capacidade orientadora da PNAF sobre políticas setoriais voltadas ao acesso da população aos medicamentos, com redução dos tempos para a sua disponibilização no SUS, redução de custos e enfretamento das vulnerabilidades do SUS. Todavia, a partir de 2014 não foram identificadas evoluções importantes nesse contexto.

Embora o período do estudo tenha estabelecido como limite o ano de 2019, tornou-se essencial a referência à situação atual de pandemia de COVID-19 a qual evidencia que investimentos no campo do desenvolvimento científico e tecnológico, bem como a adoção de marcos e novas práticas regulatórias são fundamentais para o país. Do mesmo modo, impõe-se ao Brasil a necessária superação de lacunas geradas no contexto das políticas setoriais, com retomada e potencialização dos avanços conquistados. Para tanto, entende-se como essencial a observância dos princípios definidos na política nacional de saúde e do seu controle social, o fortalecimento do sistema nacional de ciência, tecnologia e inovação e a preservação das empresas estatais com impactos no campo da saúde, tendo o Estado como regulador capaz de priorizar o desenvolvimento nacional, ao contrário de subordina-lo aos interesses econômicos particulares ou ao descaso institucional.

\section{Colaboradores}

N Rech e MR Farias trabalharam na concepção e na redação final do artigo. 


\section{Referências}

1. Brasil. Conselho Nacional de Saúde. Resolução nº 338, de 6 de maio de 2004. Aprova a Política Nacional de Assistência Farmacêutica. Diário Oficial da União 2004; 20 maio.

2. Gadelha CAG. O complexo industrial da saúde e a necessidade de um enfoque dinâmico na economia da saúde. Cien Saude Colet 2003; 8(2):521-535.

3. Gadelha CAG, Costa LS, Maldonado J. O Complexo Econômico-Industrial da Saúde e a dimensão social e econômica do desenvolvimento. Rev Saude Publica 2012; 46(Supl.):21-28.

4. Gadelha CAG, Temporão JG. Desenvolvimento, Inovação e Saúde: a perspectiva teórica e política do Complexo Econômico-Industrial da Saúde. Cien Saude Colet 2018; 23(6):1891-1902.

5. Barbui C, Addis A, Amato L, Traversa G, Garattini S. Can systematic reviews contribute to regulatory decisions? Eur J Clin Pharmacol 2017; 73:507-509.

6. Spindler P, Bach KF, Schmiegelow M, Bedlington N, Eichler HG. Innovation of Medical Products: The Evolution of Regulatory Science, Research, and Education. Ther Innov Regul Sci 2016; 50(1):44-48.

7. Tsukamoto K, Takenaka T. Role of Academia in Regulatory Science for Global Drug Development. Yakugaku Zasshi 2016; 136(4):543-547.

8. Baldwin R, Black J, O'leary G. Risk Regulation and Transnationality: Institutional Accountability as a Driver of Innovation. Transnational Environmental Law 2014; 3(2):373-390.

9. Lucchese G. Globalização e Regulação Sanitária: Os Rumos da Vigilância Sanitária no Brasil. [tese] Rio de Janeiro: Escola Nacional de Saúde Pública Sergio Arouca, Fundação Oswaldo Cruz; 2001.

10. Silva APJ, Tagliari, POP. Iniciativas de convergência regulatória em saúde nas Américas: histórico, evolução e novos desafios. Rev Panam Salud Publica 2016; 39(5):281-287.

11. Allchurch MH, Barbano DBA, Pinheiro MH, Lazdin -Helds J. Fifty years of the European medicines regulatory network: reflections for strengthening intra-regional cooperation in the Region of the Americas. Rev Panam Salud Publica 2016; 39(5):288-293.

12. World Health Organization (WHO). Member States. [acessado 2018 nov 16]. Disponível em: http://www. who.int/countries/en/.

13. Mendonça CS, Reis AT, Moraes JC, organizadores. A política de regulação do Brasil. Brasília: Organização Pan-Americana da Saúde; 2006.

14. Silva JAA, Costa EA, Lucchese G. SUS 30 anos: Vigilância Sanitária. Cien Saude Colet 2018; 23(6):19531961.

15. Meyer RJ. The Role of Academic Medical Centers in Advancing Regulatory Science. Clin Pharmacol Ther 2014; 95(51):29-31.

16. Tominaga T, Asahina Y, Uyama Y, Kondo T. Regulatory Science as a bridge between Science and society. Clin Pharmacol Ther 2011; 90(1):29-31.

17. Jasanoff $S$. Procedural choices in regulatory science. Technol Soc 1995; 17(3):279-293.

18. Food and Drug Administration (FDA). Advancing Regulatory Science at FDA. 2011. [cited 2018 Set 24]. Available from: https://www.fda.gov/downloads/ ScienceResearch/SpecialTopics/RegulatoryScience/ UCM268225.pdf.
19. Kurz X. Translating regulatory science into better processes. Eight Stakeholders forum on the implementation of the Pharmacovigilance legislation: Building on two years of operation. London: European Medicines Agency; 2018.

20. Walt G, Gilson L. Reforming the health sector in developing countries: the central role of policy analysis. Health Policy Plan 1994; 9(4):353-370.

21. Matus C. Política, planejamento e governo. Brasília: Instituto de Pesquisa Econômica Aplicada; 1993.

22. Buse K. Addressing the theoretical, practical and ethical challenges inherent in prospective health policy analysis. Health Policy and Planning 2008; 23:351-360.

23. Walt G, Shiffman J, Schneider H, Murray S, Brugha R, Gilson L. Doing' health policy analysis: methodological and conceptual reflections and challenges. Health Policy Plan 2008; 23:308-317.

24. Muller P, Surel Y. Análise das políticas públicas. Pelotas: EDUCAT; 2002.

25. Rech N. Acesso aos medicamentos e à assistência farmacêutica. Brasília: Ministério da Saúde, Secretaria de Ciência, Tecnologia e Insumos Estratégicos; 2005.

26. Rech N, Farias MR. Assistência Farmacêutica, intersetorialidade, pesquisa e inovação. Newsletter $A B C F$ 2015; 1:7-9.

27. Bermudez JAZ, Esher A, Osorio-de-Castro CGS, Vasconcelos DMM, Chaves GC, Oliveira MA, Silva, RM, Luiza VL. Assistência Farmacêutica nos 30 anos do SUS na perspectiva da integralidade. Cien Saude Colet 2018; 23(6):1937-1951.

28. Brasil. Ministério da Saúde (MS). Conselho Nacional de Saúde. $1^{a}$ Conferência Nacional de Medicamentos e Assistência Farmacêutica. Relatório Final: efetivando o acesso, a qualidade e a humanização na assistência farmacêutica, com controle social. Brasília: MS; 2005.

29. Brasil. Presidência da República. Decreto de 12 de novembro de 1991. Delega competência ao Ministro de Estado da Saúde para homologar as decisões do Conselho Nacional de Saúde. Diário Oficial da União 1991; 13 nov.

30. Leite SN, Manzini F, Veiga A, Lima MEO, Pereira MA, Araujo, SQ, Santos RF, Bermudez JAZ. Ciência, Tecnologia e Assistência Farmacêutica em pauta: contribuições da sociedade para a $16^{\mathrm{a}}$ Conferência Nacional de Saúde. Cien Saude Colet 2018; 23(12):4259-4268.

31. Brasil. Ministério da Saúde (MS). Ministério do Desenvolvimento, Indústria e Comércio Exterior. Fórum de competitividade da cadeia produtiva farmacêutica 2003-2006: O desafio de prosseguir Brasília: MS; 2007.

32. Brasil. Casa Civil da Presidência da República. Diretrizes de politica industrial, tecnológica e de comércio exterior. Brasília: Casa Civil da Presidência da República; 2004.

33. Brasil. Agência Brasileira de Desenvolvimento Industrial (ABDI). Dez anos de política industrial: balanço e perspectivas 2004-2014. Brasília: ABDI; 2015.

34. Palmeira Filho PL, Pieroni JP, Antunes MAS, Martins JV. O desafio do financiamento à inovação farmacêutica no Brasil: a experiência do BNDES Profarma. Revista do BNDES 2012; 37:67-90. [acessado 2019 maio 20]. Disponível em: http://www.bndes.gov.br/bibliotecadigital. 
35. Goldbaum M, Serruya SJ. O Ministério da Saúde na política de ciência, tecnologia e inovação em saúde. REVISTA USP 2007; 73:40-47.

36. Guimarães R, Noronha J, Elias FTS, Gadelha CAG, Carvalheiro JR, Ribeiro A. Política de ciência, Tecnologia e Inovação em Saúde. Cien Saude Colet 2019; 24(3):881-886.

37. Brasil. Conselho Nacional de Saúde. Ministério da Saúde (MS). Secretaria de Ciência, Tecnologia e Insumos Estratégicos. $2^{a}$ Conferência Nacional de Ciência, Tecnologia e Inovação em Saúde. Brasília: MS; 2005.

38. Tojal SBB, Pessôa PR. Anuência prévia na concessão de patentes de medicamentos e a regulação econômica da indústria farmacêutica. Revista de Direito Sanitário 2008; 8(3):148-165.

39. Brasil. Agência Nacional de Vigilância Sanitária. Instituto Nacional de Propriedade Industrial. Portaria Conjunta $n^{\circ} 1$, de 12 de abril de 2017. Regulamenta os procedimentos para a aplicação do artigo $229-\mathrm{C}$ da Lei no 9.279, de14 de maio de 1996, acrescido pela Lei $\mathrm{n}^{\circ} 10.196$, de 14 de fevereiro de 2001, e dá outras providências. Diário Oficial da União 2017: 13 abr.

40. Brasil. Ministério da Saúde (MS). Portaria $n^{\circ} 886$, de 24 de abril de 2007. Declara de interesse público os direitos de patente sobre o efavirenz, para fins de concessão de licença compulsória para uso não comercial. Diário Oficial da União 2007; 25 abril.

41. Brasil. Presidência da República. Decreto ${ }^{\circ} 6.108$ de 4 de maio de 2007. Concede licenciamento compulsório, por interesse público, de patentes referentes ao Efavirenz, para fins de uso público não-comercial. $D i$ ário Oficial da União 2007; 5 maio.

42. Bermudez JAZ, Oliveira MA, Esher A. Acesso a Medicamentos: Derecho Fundamental, Papel del Estado. Rio de Janeiro: ENSP; 2004.

43. Granjeiro A, Laurindo da Silva L, Teixeira PR. Resposta à aids no Brasil: contribuições dos movimentos sociais e da reforma sanitária. Rev Panam Salud Publica 2009; 26(1):87-94.

44. Hoirisch C. Licença Compulsória para Medicamentos como Política Pública: O Caso do Antirretroviral Efavirenz [dissertação]. Rio de Janeiro: Fundação Getúlio Vargas; 2010.

45. Brasil. Lei ${ }^{\circ} 9.313$ de 13 de novembro de 1996. Dispõe sobre a distribuição gratuita de medicamentos aos portadores do HIV e doentes de AIDS. Diário Oficial da União 1996; 14 nov.

46. Brasil. Presidência da República. Lei n ${ }^{\circ} 9.279$, de 14 de maio de 1996. Regula direitos e obrigações relativos à propriedade industrial. Diário Oficial da União 1996; 15 mai.

47. Greco DB. Desafios da Epidemia de Aids após 10 anos de terapia antirretroviral. Brasília: Ministério da Saúde; 2007.

48. Rodrigues WCV, Soler O. Licença compulsória do efavirenz no Brasil em 2007: contextualização. $\mathrm{Rev} \mathrm{Pa}$ nam Salud Publica 2009; 26(6):553-559.

49. Brasil. Ministério do Planejamento, Orçamento e Gestão, Ministério da Saúde, Ministério de Ciência, Tecnologia e Inovação. Portaria n ${ }^{\circ} 128$ de 29 de maio de 2008. Estabelece as diretrizes para a contratação de fármacos e medicamentos pelos órgãos e entidades integrantes do Sistema Único de Saúde. Diário Oficial da União 2008; 30 mai.
50. Viana ALD, Silva HP, Ibañez N, Iozzi FL. A política de desenvolvimento produtivo da saúde e a capacitação dos laboratórios públicos nacionais. Cad Saude Publica [periódico na Internet]. 2016 Nov [acessado 2019 maio 12]; 32(Supl. 2): [cerca de 14 p.]. Disponível em: http://www.scielo.br/scielo.php?script=sci_arttext\&pid $=$ S0102=311-2016001405003X\&lng=en\&nrmiso.

51. Brasil. Agência Nacional de Vigilância Sanitária. Portaria $n^{\circ} 583$ de 8 de agosto de 2007. Institui o Comitê Técnico-Regulatório no âmbito da Anvisa com a finalidade de acompanhar o processo de desenvolvimento, produção e registro no Brasil do medicamento objeto do Decreto n. 6.108, de 04 de maio de 2007. Diário Oficial da União 2007; 08 ago.

52. Brasil. Fundação Oswaldo Cruz (Fiocruz). Farmanguinhos entrega ao Ministério da Saúde o Efavirenz nacional. Rio de Janeiro: Fiocruz; 2009.

53. Vargas M, Gadelha CAG, Costa LS, Maldonado J. Inovação na indústria química e biotecnológia em saúde: em busca de uma agenda virtuosa. Rev Saude Publica 2012; 46(Supl.):37-40.

54. Brasil. Ministério da Saúde (MS). Mais Saúde: direito de todos 2008-2011. Brasília: MS; 2008.

55. Brasil. Decreto de 12 de maio de 2008. Cria, no âmbito do Ministério da Saúde, o Grupo Executivo do Complexo Industrial da Saúde - GECIS e dá outras providências. Diário Oficial da União 2008; 13 mai.

56. Vargas MA, Almeida ACS, Guimarães ALC. Parcerias para desenvolvimento produtivo (PDPS-MS): contexto atual, impactos no sistema de saúde e perspectivas para a política industrial. Rio de Janeiro: Fundação Oswaldo Cruz; 2017.

57. Departamento Intersindical de Estatística e Estudos Socioeconômicos (DIEESE). Política de desenvolvimento produtivo - nova política industrial do governo. São Paulo: DIEESE; 2008.

58. Brasil. Ministério da Saúde (MS). Portaria $n^{\circ} 1.942$ de 17 de setembro de 2008. Aprova o Regimento Interno do Grupo Executivo do Complexo Industrial da Saúde - GECIS e institui o Fórum Permanente de Articulação com a Sociedade Civil. Diário Oficial da União 2008; 18 set.

59. Gomes R, Pimentel Vitor, Losada M, Pieroni JP. O novo cenário de concorrência na indústria farmacêutica brasileira. BNDES Setorial 2014; 39:97-134.

60. Cassiolato JE, Szapiro M. Os dilemas da política industrial e de inovação: os problemas da região Sudeste são os problemas do Brasil. In: Leal CLCF, Linhares LRF, Lemos CR,Silva MML,Lastres HMM. Um olhar territorial sobre o desenvolvimento: Sudeste. Rio de Janeiro: BNDES; 2015.

61. Silva GO, Elias FTS. Parcerias para o desenvolvimento produtivo: um estudo de avaliabilidade. Com Cienc Saude 2017; 28(2):125-139.

62. Varrichio PC. As parcerias para o desenvolvimento produtivo da saúde. In: Rauen AT (organizador). Políticas de inovação pelo lado da demanda no Brasil. Brasília: Ipea; 2017. p.179-234.

63. Silva GO, Rezende KS. Parcerias para o desenvolvimento produtivo: a constituição de redes sociotécnicas no Complexo Econômico-Industrial da Saúde. Vigil Sanit Debate 2017; 5(1):11-12. 
64. Gadelha CAG, Braga PSC. Health and innovation: economic dynamics and Welfare State in Brazil. Cad Saude Publica 2016; 32(Supl. 2):e00150115.

65. Brasil. Agência Nacional de Vigilância Sanitária. Resolução de Diretoria Colegiada - RDC - n 2 de 2 de fevereiro de 2011. Dispõe sobre os procedimentos no âmbito da ANVISA para acompanhamento, instrução e análise dos processos de registro e pós-registro, no Brasil, de medicamentos produzidos mediante parcerias público-público ou público- privado e transferência de tecnologia de interesse do Sistema Único de Saúde. Diário Oficial da União 2011; 3 fev.

66. Brasil. Presidência da República. Decreto nº 7.540 de e de agosto de 2011. Institui o Plano Brasil Maior PBM e cria o seu sistema de gestão. Diário oficial da União 2011; 3 ago.

67. Bercovici G. Complexo industrial da saúde, desenvolvimento e proteção constitucional ao mercado interno. Rev Dir Sanit 2013; 14(2):9-42.

68. Brasil. Ministério da Saúde (MS). Portaria n 506 de 21 de março de 2012. Institui o Programa para o Desenvolvimento do Complexo Industrial da Saúde (PROCIS) e seu Comitê Gestor. Diário Oficial da União 2012; 22 mar.

69. Brasil. Ministério da Saúde (MS). Portaria n ${ }^{\circ} 837$ de 18 de abril de 2012. Define as diretrizes e os critérios para o estabelecimento as Parcerias Para o Desenvolvimento Produtivo (PDP). Diário Oficial da União 2012; 27 abr.

70. Brasil. Ministério da Saúde (MS). Plano Nacional de Saúde (PNS) 2012-2015. Brasília: MS; 2011.

71. Brasília. Agência Nacional de Vigilância Sanitária. Resolução da Diretoria Colegiada RDC - nº 50 de 13 de setembro de 2012. Dispõe sobre os procedimentos no âmbito da Anvisa para registro de produtos em processo de desenvolvimento ou de transferência de tecnologias objetos de Parcerias de Desenvolvimento Produtivo público-público ou público-privado de interesse do Sistema Único de Saúde. Diário Oficial da União 2012; 14 set.

72. Brasil. Ministério da Saúde (MS). Portaria GM/MS n ${ }^{\circ}$ 2.531 de 12 de novembro de 2014. Redefine as diretrizes e os critérios para a definição da lista de produtos estratégicos para o Sistema Único de Saúde (SUS) e o estabelecimento das Parcerias para o Desenvolvimento Produtivo (PDP) e disciplina os respectivos processos de submissão, instrução, decisão, transferência e absorção de tecnologia, aquisição de produtos estratégicos para o SUS no âmbito das PDP e o respectivo monitoramento e avaliação. Diário Oficial da União 2014; 13 nov.
73. Oliveira EJV, Vivan AL, Albuquerque FC, Silva GO, Rezende KS, Barbosa LP. A consolidação do marco regulatório das parcerias para o desenvolvimento produtivo. Revista Eletrônica Gestão em Saúde [periódico na Internet]. 2015 out [acessado 2016 mar 18]; 6(Supl. 4): [cerca 19 p.]. Disponível em: blob:https:// periodicos.unb.br/7473880f-c9af-4da8-b0a1-3351a6 ec1472

74. Brasil. Presidência da República. Decreto nº 9.245 de 20 de dezembro de 2017. Institui a Politica Nacional de Inovação Tecnológica na Saúde. Diário Oficial da União 2017; 21 Dez.

75. Souza LEPF, Paim JS, Teixeira CF, Bahia L, Guimarães R, Machado CV, Campos GW, Azevedo-e-Silva G. Os desafios atuais da luta pelo direito universal à saúde no Brasil. Cien Saude Colet 2019; 24(8):2783-2792.

76. Brasil. Tribunal de Contas da União. Sessão Extraordinária Reservada do Plenário - Ata 18, de 9 de agosto de 2017. Brasília: Secretaria da Sessões/TCU, 2017. Disponível em: http://www.tcu.gov.br/Consultas/Juris/ Docs/CONSES/TCU_ATA_0_S_2017_18.PDF

77. Brasil. Ministério da Saúde (MS). Portaria no 978 de 16 de maio de 2008. Dispõe sobre a lista de produtos estratégicos, no âmbito do Sistema Único de Saúde, com a finalidade de colaborar com o desenvolvimento do Complexo Industrial da Saúde e institui a Comissão para Revisão e Atualização da referida lista. Diário Oficial da União 2008; 17 mai.

78. Rezende KS, Silva GO, Albuquerque FC. Parcerias para o Desenvolvimento Produtivo: um ensaio sobre a construção das listas de produtos estratégicos. Saude Debate 2019; 43(2):155-168.

79. Silva GO, Elias FTS. Parcerias para o Desenvolvimento Produtivo: diagnóstico situacional da implementação na perspectiva dos atores envolvidos. Com Cienc Saude 2017; 28(3/4):313-325.

Artigo apresentado em 24/03/2021

Aprovado em 02/08/2021

Versão final apresentada em 04/08/2021

Editores-chefes: Romeu Gomes, Antônio Augusto Moura da Silva 